\title{
SIMULATION OF THE RECYCLER RING DYNAMIC APERTURE
}

\author{
C. S. Mishra ${ }^{+}$and D. Johnson \\ Fermi National Accelerator Laboratory* \\ Batavia, IL 60510
}

\begin{abstract}
The Fermilab Recycler Ring is being built at Fermilab to support the Run-II and beyond physics goals of Fermilab. The role of the Recycler Ring is to provide more antiprotons for the Tevatron, which proportionally increases the luminosity. This paper will describe the Recycler Ring Lattice design and simulations of the dynamic aperture using data from the some of the magnets installed in the ring.
\end{abstract}

\section{INTRODUCTION}

The Main Injector, a rapid cycle $150 \mathrm{GeV}$ proton synchrotron, will supply a larger flux of protons for antiproton production; more intense proton bunches for use in the collider, and higher efficiency acceleration for both proton and antiprotons. The role of the Recycler Ring [1] is to provide more antiprotons for the Tevatron, which proportionally increases the luminosity. Recycler accomplishes this by acting as a high reliability postAccumulator and receptacle for recycled antiprotons from previous Collider stores. Prior to the development of the Recycler ring, the peak luminosity goal of the Fermi III upgrade program was $8 \times 10^{31} \mathrm{~cm}^{-2} \mathrm{sec}^{-1}$. With the construction of the Recycler ring, a typical peak luminosity of $2 \times 10^{32} \mathrm{~cm}^{-2} \mathrm{sec}^{-1}$ is expected The Recycler is also the foundation of future accelerator upgrades that can increase the Tevatron Luminosity up to $1 \times 10^{33} \mathrm{~cm}^{-2} \mathrm{sec}^{-1}$.

The Recycler is a fixed $8 \mathrm{GeV}$ kinetic energy storage ring made up of permanent magnets. This Recycler ring lattice mimics closely the Main Injector lattice. The ring was designed using MAD [2] such that it replicated the Main Injector cell length and hence followed the footprint of the Main Injector. This paper describes the lattice design of the Recycler ring. Simulation results of the Recycler performance using data from initial gradient magnets are presented in this paper. The Recycler Lattice includes the magnetic field errors, both systematic and random, and misalignment errors. Studies of dynamic aperture and momentum aperture are presented in this paper. Results shows that the simulated dynamic aperture using realistic magnet and alignment information meets the design specification of $40 \pi \mathrm{mm}-\mathrm{mr}$. The production magnets are of better quality as compared to the design specification [3]. A thin element tracking program TEAPOT [4] has been used for these simulations.

\section{LATTICE DESIGN}

The lattice is a strong focusing FODO lattice made up of either two gradient magnets or two quadrupoles. The Recycler ring is composed of fifty-four arc FODO cells (17.288 meter cell length) with eight zero dispersion straight sections distributed around the ring following the symmetry of the new Main Injector. The lattice has been design to have a base tune of $\mathrm{Q}_{\mathrm{x}}=24.425$ and $\mathrm{Q}_{\mathrm{y}}=$ 24.415 with a maximum horizontal dispersion of $2 \mathrm{~m}$ and a corrected chromaticity of -2 units in each plane. The phase advance per cell is $\phi_{\mathrm{x}}=85.4 \mathrm{deg}$ and $\phi_{\mathrm{y}}=79.2 \mathrm{deg}$. The phase advance across high beta insert at MI-30 makes the integer part of the $\mathrm{x}$ and $\mathrm{y}$ tunes same.

The Recycler lattice is virtually indistinguishable from the Main Injector lattice with two exceptions. The one exception is at MI-60, the phase trombone sections and the other is at MI-30, the high beta insert for electron cooling. The phase trombone at MI-60 is used to adjust the tune variation caused by magnet imperfections.

The Ring is composed of two types of permanent magnet combined function magnets (CFM), 216 long "arc" magnets which have dipole, quadrupole and sextupole components, and 128 short "dispersion suppressor" magnets which only have dipole and quadrupole components, and 74 permanent magnet quadrupoles. The Recycler ring is designed to match the momentum of the new permanent magnet $8 \mathrm{GeV}$ injection line.

In an earlier paper [3] we had outlined the magnetic field specifications for the Recycler Magnet. To date all the magnets have been build and installed in the ring. The simulations presented in this paper were performed at the

\footnotetext{
+Email:mishra@fnal.gov

* Operated by University Research Association under contract with the U.S. Department of Energy.
} 
time when first 24 CFM's were build and its higher multipoles trimmed to the specification. Table 1. Shows the multipoles used in the simulations. All of these multipoles are better than specification. Final magnet data not used in this simulation is slightly better than these values. We have used a mean of 0.1 units and sigma of 0.25 units for pole 6 to 9 . The quadrupole multipole data

\begin{tabular}{|l|l|l|l|l|}
\hline & Normal & Normal & Skew & Skew \\
\hline Pole & Mean & Sigma & Mean & Sigma \\
\hline 0 & 0.56 & 3.78 & - & - \\
\hline 1 & 0.20 & 0.65 & 0.06 & 1.56 \\
\hline 2 & -0.27 & 0.31 & -0.15 & 0.42 \\
\hline 3 & 0.0 & 0.28 & 0.06 & 0.37 \\
\hline 4 & -0.07 & 0.19 & 0.13 & 0.25 \\
\hline 5 & -0.01 & 0.22 & 0.01 & 0.17 \\
\hline
\end{tabular}

Table 1. Combined Function Magnet data used in the Simulation.

was not available at the time of these simulations. We continued to use the multipoles of 0.5 units mean and 1.5 Units sigma. The calculations also includes a misalignment errors $\sigma_{\mathrm{x}}=\sigma_{\mathrm{y}}=0.25 \mathrm{~m}$ and $\sigma_{\text {roll }}=0.5 \mathrm{mrad}$ for all magnetic elements and BPMs.

\section{DYNAMIC APERTURE}

In this paper we describe the variation of magnet properties from ideal and their effect on dynamic aperture of the Recycler. Finally a simulation where all the effects are considered together is discussed. The measured longitudinal profile of the magnetic fields $b 0, b 1$ and $b 2$ was found to have a shape different than parabolic, as assumed in earlier calculations. In these calculations we use an average of $20 \mathrm{CFMs}$ longitudinal profile data to simulate the longitudinal b0, b1 and b2 variations. Dividing the CFM into 16 magnets simulates magnet profile. Each individual piece has its own deviations from the nominal. Figure 1., shows the longitudinal profiles used in simulations at various times. The three-bump profile (2) is from the magnet data. We have found that the longitudinal profile variations do not have much effect on the dynamic aperture.

Launching an array of particles at different amplitudes tests performance in the presence of a mixture of alignment and magnetic field error. Particles are tracked with betatron oscillation amplitudes relative to a corrected orbit. Following the introduction of errors the tune is adjusted to the nominal tune for zero momentum offset using the phase trombone. The particles are launched with equal horizontal and vertical emittance. Particles are tracked with a constant momentum offset. Unless otherwise specified the momentum offset for these simulations are $0.3 \%$. Net chromaticities in both planes are set to -2 . Simulations are performed for five seed fir $1 \mathrm{e} 5$ to $1 \mathrm{e} 6$ turns corresponding to a maximum of 10 -sec store time.

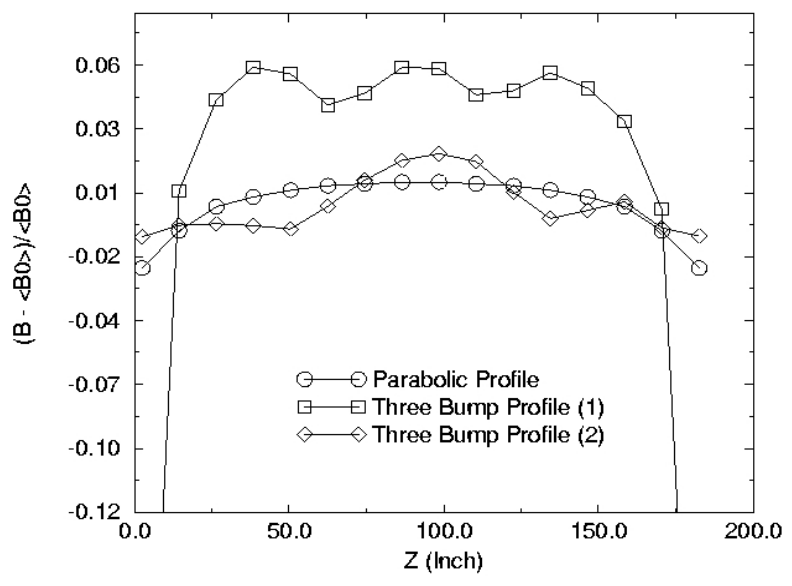

Figure 1. Longitudinal profiles of the CFMs.

As described earlier the "acr" CFM has sextupole component build into its body field. The Recycler Lattice has two families of distributed sextupoles correctors with enough strength to adjust the chromaticity by 10 units. There are 8 and 16 sextupoles in the $\mathrm{H}$ and $\mathrm{V}$ planes respectively, distributed around the ring. These correctors will compensate any change in chromaticity values due to any systematic sextupole offset from the design. During the construction of the arc CFM it was found that the body sextupole and other multipoles were different than the design specifications. The CFM body multipole components were trimmed by placing a specially designed multipole trim for that particular magnet at one end of the CFM. These trims also contained a component for systematic sextupole offset. We have simulated the effect of these end sextupole trims on the dynamic aperture of the Recycler. Any change in chromaticity due to this oneended correction was adjusted by using the distributed

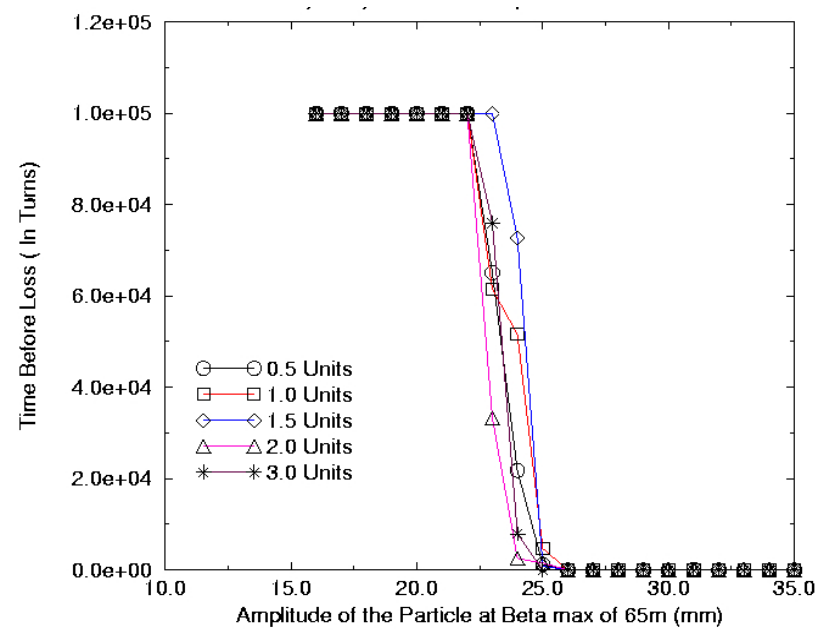


Figure 2. Dynamic Aperture for different size of end trims sextupole.

correction sextupoles. Figure 2. shows that for a 1 second simulation of the Recycler the dynamic aperture is independent of the size of the end trim sextupole.

A survival plot of the five seeds displaying how many turns a particle survived as a function of the initial launch amplitude is shown in Figure 3. This calculation included the longitudinal profile, the feed-down of higher multipoles due to curvature, measured magnetic multipoles, end shim sextupoles of 3 units. The dynamic aperture of the machine is defined as the smallest amplitude particle that did not survive the full 1e6 turns. From figure 3 we see that the dynamic aperture of the Recycler is predicted to be $24.2+/-1.5 \mathrm{~mm}$ corresponding to a normalized emittance of $78.9+/-10 \pi \mathrm{mm}-\mathrm{mr}$.

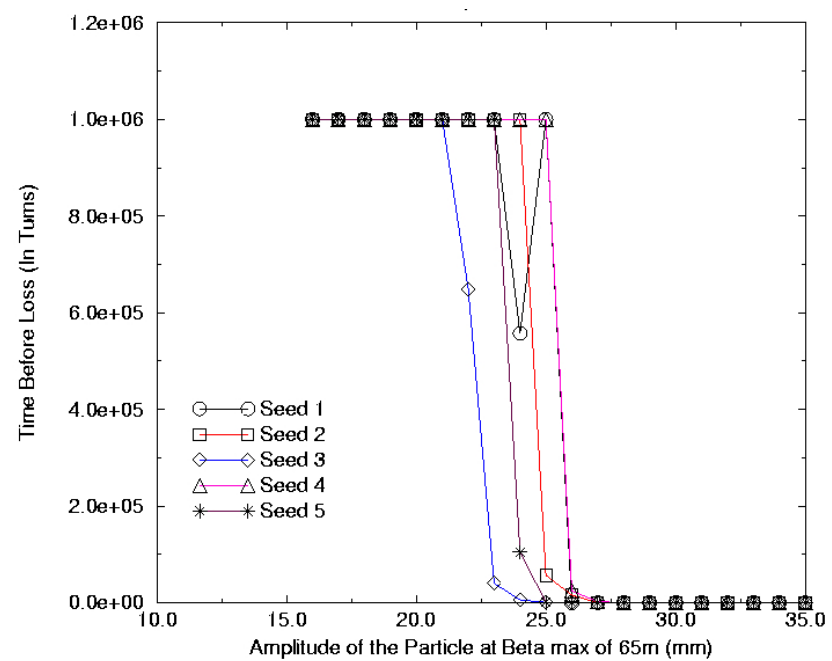

Figure 3: Dynamic Aperture of the Recycler. The number of turns survived is shown as a function of launch amplitude for five different collections of systematic and random alignment and magnetic field errors and a momentum offset of $0.3 \%$.

The dynamic aperture as a function of momentum offset for the largest amplitude particles that survived 1e5 turns

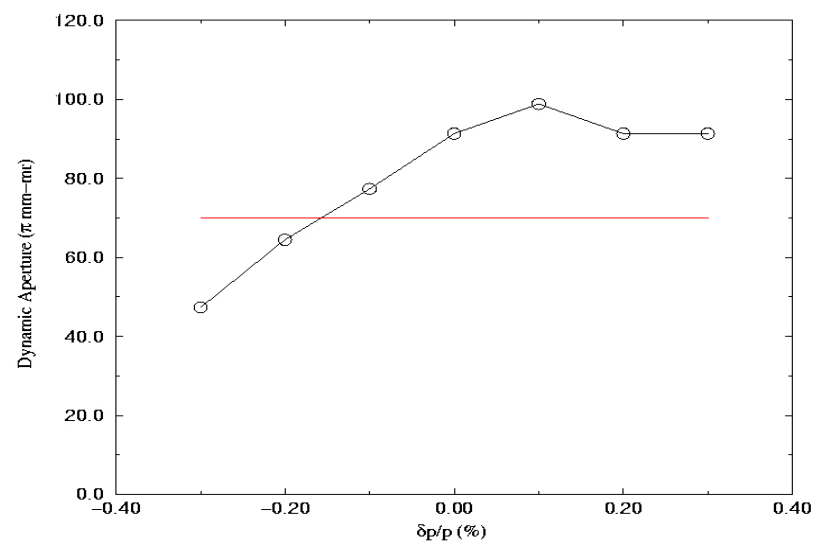

Figure 4: Dynamic Aperture as a function of momentum offset over the full range of the antiproton stack.

is shown in Figure 4. This plot indicates an aperture in excess of $60 \pi \mathrm{mm}-\mathrm{mr}$ is maintained over the full momentum spread expected in the antiproton stack. Loss in aperture for negative $\delta \mathrm{p} / \mathrm{p}$ indicates that the particles are moving towards a resonance. In principle this can be improved by small adjustment in the operating tune.

\section{SUMMARY}

The design criteria of the Recycler was that the dynamic aperture calculated based on 10 seconds simulation of the Recycler should be as large as the physical aperture. Also there should be no trend of particle loss. All the Recycler magnets have build, sorted and installed in the ring. The magnet data in most cases are better than specified. The commissioning of the Recycler ring is going to start during April 1999.

\section{REFERENCE}

[1] "The Fermilab Main Injector Technical Design Handbook", August 1994.

[2] "The Fermilab Recycler Ring Technical Design Report", Fermilab TM-1991, November 1996.

[3] MAD, An Acceleration Design Code, CERN, Geneva.

[4] D. E. Johnson et al.," Design and Simulation of the Antiproton Recycler Lattice", Proceeding of PAC97.

[5] L. Schachinger and R. Talman, Particle Accelerators 22, 1987.

[6] Al Garren and Dave Robin, "A Phase Trombone for the Recycler", internal MI note MI-0181, July 1995. 